\title{
Influence of Embedded Microprocessor Wireless Communication and Computer Vision in Wushu Competition Referees' Decision Support
}

\author{
Jingjing Ji ${ }^{1}$ and Feng Liang $\mathbb{D}^{2,3}$ \\ ${ }^{1}$ Department of Physical Education, Southeast University, Nanjing, 211189 Jiangsu, China \\ ${ }^{2}$ Guilin Tourism University, Guili, 541000 Guangxi, China \\ ${ }^{3}$ Institute of Sports Economic Theory, East China University of Science and Technology, Shanghai 200237, China \\ Correspondence should be addressed to Feng Liang; lf@gltu.edu.cn
}

Received 28 September 2021; Revised 18 November 2021; Accepted 24 November 2021; Published 17 January 2022

Academic Editor: Haibin Lv

Copyright (C) 2022 Jinging Ji and Feng Liang. This is an open access article distributed under the Creative Commons Attribution License, which permits unrestricted use, distribution, and reproduction in any medium, provided the original work is properly cited.

\begin{abstract}
With the development of computer technology and management science, decision support systems have emerged that can improve the quality and effects of decision-making. This study mainly examined the application of the wireless communication of an embedded microprocessor and computer vision in the decision support system of martial arts competition referees. Using the embedded microprocessor's characteristics of a small size, high precision, high reliability, and high efficiency, a decision support system for martial arts competition referees was designed. In the experiment, the similarity between the target field and the source field could be controlled by adjusting the mean value. To better extract the target, this study used the time domain changes of the three adjacent frames, before, middle, and back, to detect the moving target to extract the change detection template. The Canny edge detection method was used to extract the edge information of the image and eliminate the nonmotion area; then, morphology was used to correct the image to complete the connection of the broken edge to obtain the final initial segmentation mask image. In the process of calculation, there were some noises and small fragments. In this study, morphology and background difference were used to optimize the segmented image. Experimental data show that the algorithm detection accuracy rate was high-between $70 \%$ and $100 \%$ - and the effect was relatively ideal. The results indicate that the proposed algorithm can effectively reduce matching noise, improve the matching accuracy of the edge area and the low-texture area, and achieve a fast matching speed.
\end{abstract}

\section{Introduction}

After a long process, human understanding of vision has changed from perceptual knowledge to qualitative and quantitative analysis. In present-day China, the most effective means of protection is to carry out the official traditional martial arts competition every year. The competition encourages the development of traditional martial arts, including statistical analyses on the participants and scale of the traditional martial arts competition in Beijing in recent years, which gives traditional martial arts a base to recuperate. Furthermore, the research goal of computer vision is to use cameras or other sensors and computers instead of the human eyes and brains to process one or more images, to establish an artificial intelligent vision system that can automatically obtain external information from images or multidimensional data.

Competitive martial arts is a pioneer in the world of sports. The depth and thickness of the training of reserve talents of competitive martial arts routines directly affect whether competitive martial arts routines can be sustainable and prosper in the world sports arena. The development of DW is based on the premise of the database. According to the requirements of decision-making, this study integrated and summarized the data of different formats in a traditional database, which provides a unified comprehensive data 
source for all kinds of users and not only provides technical support for accurate data analysis based on the data but also provides users with development trends and information that can be queried at any time.

The development of computer vision is accelerating. Computer vision is the science of how to make machines "see," and it refers to the use of cameras and computers instead of the human eye to identify, track, and measure targets and other machine vision, as well as to perform graphics processing to make the computer processing into images that are more suitable for observation by the human eye or transmission to instruments for testing. Tarlak et al. have proposed and demonstrated a simple and alternative method of measuring the color of food, called a "computer vision system." With the aid of the computer vision system, it is possible to quickly, inexpensively, and simply classify the color of food that is uniform and homogeneous in color and shape. The system can also be used to differentiate between defective and nondefective products [1]. Ludovico et al. developed NeuriteTracker as a computer vision method that automatically segments and tracks neuronal morphodynamics in a timelapse dataset. Feature extraction then quantifies the dynamic neuronal growth phenotype. The authors identified a stereotyped set of neuronal growth morphodynamic behaviors in a cultured neuronal cell system. Systematic RNA interference perturbations of the Rho GTPase interactome, consisting of 219 proteins, revealed a limited set of morphodynamic phenotypes. It was shown that the loss of function of two different RhoA-specific GTPaseactivating proteins (GAP) led to opposite neurite growth [2]. Cha Y J proposed a damage detection method. Compared with the traditional method, the method does not require an intensive instrumentation process, does not add any additional quality to the structure, may skew the measurement results, and can measure more signals. He used the displacement measured in the video to detect structural damage, and the tasteless Kalman filter was used to remove noise from the displacement measurement. He detected the damage at the same time by determining the current stiffness and damping coefficient values for a given known mass. However, his research is not accurate enough [3].

This study combined the movement characteristics of athletes in martial arts competitions to detect sports targets. Wireless communication technology and computer vision technology using the embedded microprocessor and the edge detection algorithm of the Canny operator were used to subtract the current frame image from the background image, thereby detecting the moving target, which provides a better method for the establishment and improvement of organizational rules such as countries, communities, and enterprises. In the process of feature extraction, owing to the change of conditions and the interference of noise during the acquisition process, the target image had angle changes or illumination changes, and the extracted texture features changed. After reordering by the saliency of the target, the accuracy of the image results returned by the search engine could be effectively improved.

\section{Decision Support System Based on Computer Vision}

2.1. Computer Vision. Assuming that an $M \times N$ image $f(x$, $y$ ) passes through an $m \times n$ ( $m$ and $n$ are odd) weighted mean filter $w(s, t)$, the processing process is

$$
g(x, y)=\frac{\sum_{s=-a}^{a} \sum_{t=-b}^{b} w(s, t) f(x+s, y+t)}{\sum_{s=-a}^{a} \sum_{t=-b}^{b} w(s, t)} .
$$

In the formula, $w(s, t)$ is the mask coefficient, and the numerator is the template coefficient and convolves with the pixel block centered on $(x, y)$. The fusion of the Bayesian principle and SVM produces RVM, whose expression is as follows:

$$
P(t \mid \lambda)=\prod_{i=1}^{n} \sigma y_{i}\left(x_{i} ; \lambda\right)^{t_{i}}\left[1-y_{i}\left(x_{i} ; \lambda\right)\right]^{1-t_{i}},
$$

where $\lambda$ is the weight vector. Low-level feature research has focused on simple and simple image objects (e.g., objects and characters), which is also the most popular field of traditional computer vision. The expression of middle-level semantic features needs to analyze the semantic information of different regions in an image [4]. The expression of middle-level semantics is more complex than that of lowlevel features. In addition to recognizing the objects in the image, it is also necessary to distinguish their position, edge, and other information accurately. The understanding of high-level semantics is oriented to the image with relatively complex content. It needs to analyze the potential semantic information of the image through the numerous objects contained in the image and then complete the "understanding" of an image at the semantic level [5]. The expression of the similarity measure function $\mathrm{SAD}$ is as follows:

$$
\operatorname{SAD}(x, y, d)=\sum_{i=-m}^{m} \sum_{j=-n}^{n}|L(x+i, y+j)-R(x+i+d, y+j)| .
$$

In the formula, $d$ is the limited parallax search range; $m$ and $n$ are the half-window size, respectively; and $L(x, y)$ and $R(x, y)$ represent the gray value at the corresponding points of the left and right images, respectively [6]. The function expressions of the similarity measurement functions SSD and NCC are as follows:

$$
\begin{gathered}
\operatorname{SSD}(x, y, d)=\sum_{i=-m}^{m} \sum_{j=-n}^{n}[L(x+i, y+j)-R(x+i+d, y+j)]^{2}, \\
\operatorname{NCC}(x, y, d)=\frac{\sum_{i=-m}^{m} \sum_{j=-n}^{n}[L(x+i, y+j)-L] \bullet[R(x+i, y+j)-R]}{\sqrt{\sum_{i=-m}^{m} \sum_{j=-n}^{n}[L(x+i, y+j)-L]^{2} \bullet[R(x+i, y+j)-R]^{2}}} .
\end{gathered}
$$

In the formula, $L$ and $R$ represent the average gray values of the left and right image neighborhoods, respectively. SAD 
and SSD measures are difference measures, and the $d$ when the result is the smallest within the limited range is used as the calculated result disparity. Moreover, the NCC measure is a similarity measure, and the $d$ when the result is the largest within the limited range is the calculated result disparity. The application scenarios of object recognition are relatively simple, mainly focusing on the extraction and classification of object features. It is a traditional field of computer vision research and one of the most in-depth research fields of convolutional neural networks [7]. Object recognition, as the basis of middle-level semantic expression and high-level semantic understanding in computer vision, has important research value. These characteristics of the samples in the object recognition data set make the research methods of object recognition relatively simple. It only needs to focus on the feature extraction and analysis of objects, avoiding background segmentation, object detection, and other disruptive tasks. Therefore, object recognition applications are usually used for feature or classifier performance evaluation [8].

Computer vision can provide a faster and simpler process by counting the execution of monotonous, repetitive tasks at a faster rate; making the whole process simpler; giving accurate results; and reducing costs. As machines take on the responsibility of performing tedious tasks, errors will be minimized, leaving no room for faulty products or services.

\subsection{Feature Extraction of Embedded Microprocessor. Feature} extraction is the visual cognitive basis of content-based image retrieval (CBIR) and the basis of image retrieval. The embedded microprocessor can better extract the features and get the result with strong processing power and a fast processing speed. The embedded microprocessor has evolved from the CPU in general-purpose computers. It is characterized by a 32-bit or higher processor with higher performance, and of course, its price is correspondingly higher. However, unlike computer processors, in practical embedded applications, only the functional hardware closely related to the embedded application is retained, and other redundant functional parts are removed, so that the special requirements of the embedded application are achieved with minimum power consumption and resources. Compared with industrial control computers, embedded microprocessors have the advantages of a small size, light weight, low cost, and high reliability. The main content of the image can be described by features, which greatly reduces the amount of calculation required for image retrieval. The features of an image include the underlying visual features, such as color, texture, and shape, and text features, such as titles, keywords, and notes. As the location of facilities and equipment in the traffic scene is fixed, the background image is relatively stable, but the overlap rate of pedestrians is high, and it is easily disturbed by black shadows [9]. The overall block diagram of the martial arts action monitoring algorithm is shown in Figure 1. The initial background is repeatedly updated in the current image frames of different sequences, and each updated background can be used as the background for the detection of moving foreground targets [10].
Edge detection based on the Canny operator needs to meet the basic criteria: (1) Signal-to-noise ratio. The edge quality extracted from the image will be higher with the improvement of the signal-to-noise ratio. (2) Unit edge response. We use the average distance of the zero-crossing point of the detection operator to meet the requirements so that there is only one response for the unit edge [11]. The specific process is as follows: (1) For the obtained image, the Gaussian filter is used for smoothing. (2) The direction and amplitude of the gradient are calculated. (3) The nonmaximum value is used to suppress the amplitude of the gradient calculated in the image. (4) The double threshold algorithm is used to detect edges and connect edges [12, 13]. The Canny operator can identify as many actual edges in the image as possible, but the identified edges should be as close as possible to the actual edges in the actual image. However, the edges in the image can only be identified once, and possible image noise should not be identified as edges.

The elements of the Sobel edge detection output image (array) usually have larger absolute values. However, as the edge is a sign of position, it is not sensitive to changes in grayscale.

The disadvantage of texture features is that they are sensitive to image resolution changes and illumination changes. For example, light reflection and shadow changes will cause texture features to change, leading to retrieval errors. To meet the requirements of automatically adapting to timefrequency signal analysis, it subdivides time at high frequencies and subdivides frequencies at low frequencies. It uses telescopic translation operations to gradually refine the signal (function) in multiple scales [14]. The wavelet basis function $\psi_{m n}(x)$ can be expressed as

$$
\psi_{m n}(x)=2^{-m / 2} \psi\left(2^{-m} x-n\right)
$$

where $m$ and $n$ are arbitrary integers. Then, the image $I$ after wavelet change is

$$
f(x)=\sum_{m, n} I_{m, n} \psi_{m n}(x)
$$

The general process of feature selection is shown in Figure 2. The goals of feature selection are to (1) remove redundant features and avoid overfitting, (2) provide a model with less computational cost, and (3) better express the potential structure information of the data. In other words, feature selection is aimed at finding the smallest possible feature subset to complete the expression task and promote the effective completion of the classification task as much as possible $[15,16]$.

The standard deviation can be used to adjust the segmentation threshold. Through this center of gravity, the central gray value moment is defined as:

$$
u_{p, q}=\frac{1}{a} \sum_{(r, c) \in R} g_{r, c}\left(r-n_{1,0}\right)^{p}\left(c-n_{0,1}\right)^{q}
$$

The energy function is defined as the sum of single point 


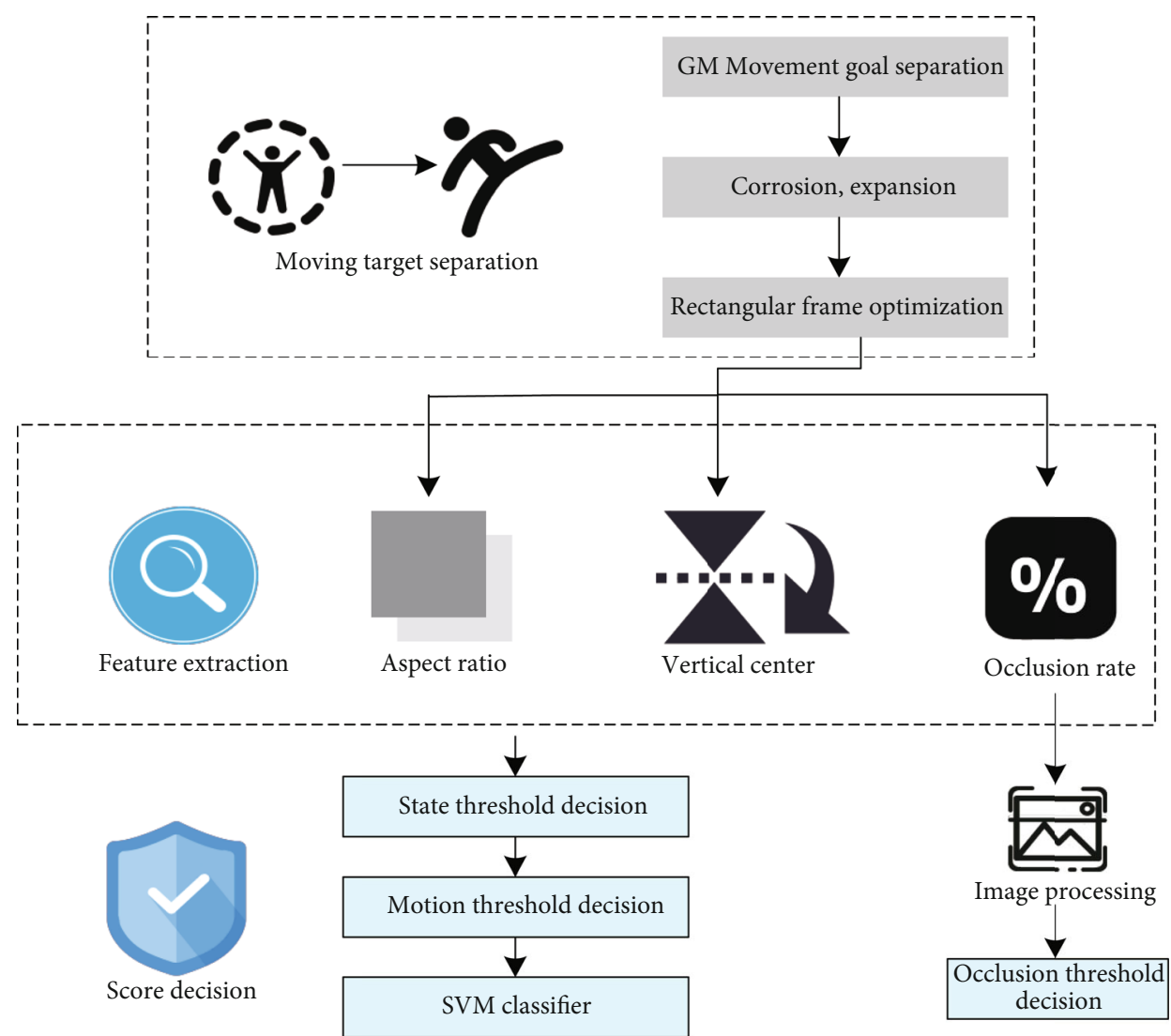

FIGURE 1: Overall block diagram of martial arts action monitoring algorithm.

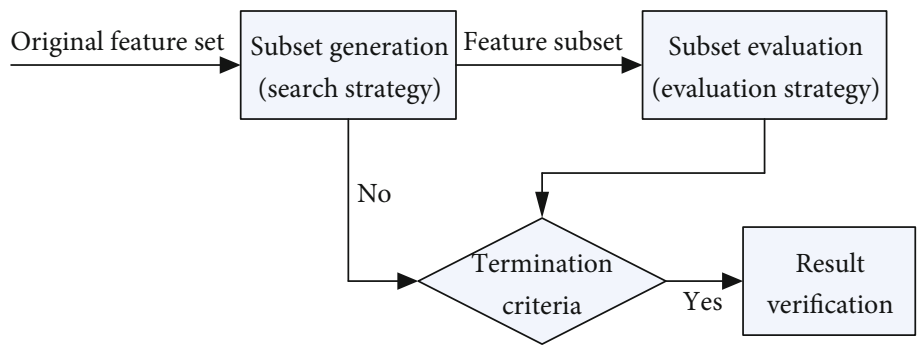

FIgURE 2: The general flow of feature selection.

potential energy and paired potential energy, and its expression is as follows:

$$
E(x)=\sum_{i \in V} \psi_{i}\left(x_{i}\right)+\sum_{i \in V, j \in N_{j}} \psi_{i j}\left(x_{i j}\right)
$$

In the formula, $N_{i}$ represents the set of all points adjacent to the variable $x_{i}$. The offset of the signal phase can be expressed by the following formula:

$$
s(x)=\sum_{0}^{n} \frac{1}{(2 n+1)^{p}} \sin [(2 n+1) x+\varphi] .
$$

Here, $p$ is the amplitude attenuation coefficient of each Fourier component, and $\varphi$ is the phase offset of the feature [17]. To evaluate the degree of phase consistency of different
Fourier components, we define phase consistency in onedimensional space as

$$
\mathrm{PC}_{1 D}(x)=\frac{|E(x)|}{\sum_{n} A_{n}(x)}=\frac{\sum_{n} A_{n}\left(\cos \left(\varphi_{n}(x)-\varphi_{\text {mean }}(x)\right)\right)}{\sum_{n} A_{n}(x)} .
$$

Among them, $|E(x)|$ is the local energy, $A_{n}$ and $\varphi_{n}$ are the magnitude and phase angle values, respectively, of the $n$-th order Fourier transform vector, and $\varphi_{\text {mean }}(x)$ is the weighted average of the local phase angles of all Fourier components at position $x$.

2.3. Decision Support System. The three-bank structure of DSS is shown in Figure 3. The structure of DSS should include the database and its management system (DBMS), 
the model library and its management system (MBMS), the method library and its management system (MEBMS), and the man-machine interface component (DGMS). The decision support system is a computer application system that helps decision-makers make semistructured or unstructured decisions through human-computer interaction through data, models, and knowledge. It is an advanced information management system produced by the development of a management information system (MIS) to a higher level. It provides decision-makers with an environment for analyzing problems, establishing models, simulating decisionmaking processes and plans, and calling various information resources and analysis tools to help decision-makers improve their decision-making level and quality. The basic structure of the decision support system is mainly composed of four parts: the data part, the model part, the reasoning part, the decision support system, and the human-computer interaction part. The data part is a database system; the model part includes the model library (MB) and its MBMS; the reasoning part is composed of the knowledge base $(\mathrm{KB})$, the knowledge base management system (KBMS), and the reasoning machine; the human-computer interaction part is the human-computer interaction interface of the decision support system, which is used to receive and verify user requests and call the internal functions of the system. The software allows for model operation, data transfer, and knowledge reasoning to be organically unified, and the decision-making problem to be solved effectively.

When the support vector machine is used for the engineering anomaly warning, the hyperplane can isolate the normal state data and abnormal state data to the maximum. The uncertainty of a single rule mainly includes the error generated when it is created, the error specified by the likelihood of the rule's antecedent, the calculation of evidence combination, and the uncertainty of the rule's consequent due to the error of the antecedent. The compilation of rules is inseparable from knowledge engineers. The subjective cognition of knowledge engineers during the compilation of rules will greatly affect the accuracy of the rules, resulting in inaccurate inference rules, unreasonable evidence combinations, and deviations in likelihood values. This results in deviations in the expert system's reasoning calculation [18]. In the particle processing industry, PBM can describe the changes of particle population characteristics over time in processes such as crystallization, granulation, crushing, and drying. The population balance model form is represented by the following formula:

$\frac{\partial V n(x, t)}{\partial t}=\dot{Q}_{\mathrm{in}} n_{\mathrm{in}}(x)-\dot{Q}_{e x} n_{e x}(x)-V \frac{\partial G n(x, t)}{\partial x}+V(\dot{b}(x)-\dot{d}(x))$,

where $x$ is the characteristic vector of particles, which can include important properties such as particle size, shape parameters, moisture content, and porosity; $n(x, t)$ is the distribution function of particle number density. The information related to particle size can be obtained by taking moments from PBM. Take the $k$-order moment of the equa- tion:

$$
\frac{d V \mu_{\mathrm{k}}^{\prime}}{d t}=\dot{Q}_{\mathrm{in}} \mu_{k, \text { in }}^{\prime}-\dot{Q}_{e x} \mu_{k, e x}^{\prime}-\int_{0}^{\infty} \frac{\partial G n(x, t)}{\partial x} d x+V \mu_{k, B}^{\prime}-V \mu_{k, D}^{\prime}
$$

If the growth rate, $G$, is independent of size, the growth term can be simplified to

$$
\int_{0}^{\infty} \frac{\partial G n(x, t)}{\partial x} d x=V G \int_{0}^{\infty} x^{k} \frac{\partial n(x, t)}{\partial x} d x=-V G k \mu_{k-1}^{\prime} .
$$

Putting this into the equation, we get

$$
\frac{d V \mu_{k}^{\prime}}{d t}=\dot{Q}_{\text {in }} \mu_{k, \text { in }}^{\prime}-\dot{Q}_{e x} \mu_{k, e x}^{\prime}+V G k \mu_{k-1}^{\prime}+V \mu_{k, B}^{\prime}-V \mu_{k, D}^{\prime}
$$

For the output layer connection weight matrix, $W^{0}$, the adjustment equation of the $p$-th row can be expressed as

$$
\Delta W_{p, k}^{0}=-\alpha \sum_{i=1}^{n_{0}} \frac{\partial \varphi\left(e_{i, k}\right)}{\partial \widehat{y}_{i, k}} \frac{\partial \sigma_{0}\left(y_{i, k}\right)}{\partial y_{i, k}} \frac{\partial y_{i, k}}{\partial W_{p}^{(0)}} .
$$

LS-SVM regression uses a quadratic penalty function; the formula is as follows:

$$
\min _{w, b, e}=J(w, e)=\frac{1}{2} w^{T} w+\frac{C}{2} \sum_{i=1}^{N} e_{i}^{2} .
$$

Among them, the former term means minimizing the VC dimension of the model, the latter term means minimizing the training error, and $C$ is the penalty coefficient. The corresponding Lagrange function is

$$
L(w, b, e, \alpha)=J(w, e)-\sum_{i=1}^{N} \alpha_{i}\left\{w^{T} \varphi\left(x_{i}\right)+b+e_{i}-y_{i}\right\}
$$

In the formula, $\alpha_{i}$ is the Lagrangian multiplier. LS-SVM only needs to determine the shape parameter of the kernel function and the penalty coefficient, $C$, and does not need to select the $\varepsilon$ value of the insensitive loss function, which not only simplifies the calculation but is also easy to use. The brittleness coefficient of the fuzzy set can be obtained by the following formula:

$$
K(x)=\frac{\sum_{i=0}^{n}\left(b_{i}-c\right)}{\sum_{i=0}^{n}\left(b_{i}-c\right)-\sum_{i=0}^{n}\left(a_{i}-d\right)} .
$$

Calculate the correlation degree between the comparison sequence and the standard sequence; the expression is as follows:

$$
\gamma\left(x_{0}, x_{i}\right)=\sum_{k=1}^{m} \beta_{k} \gamma\left(x_{0}(k), x_{i}(k)\right)
$$




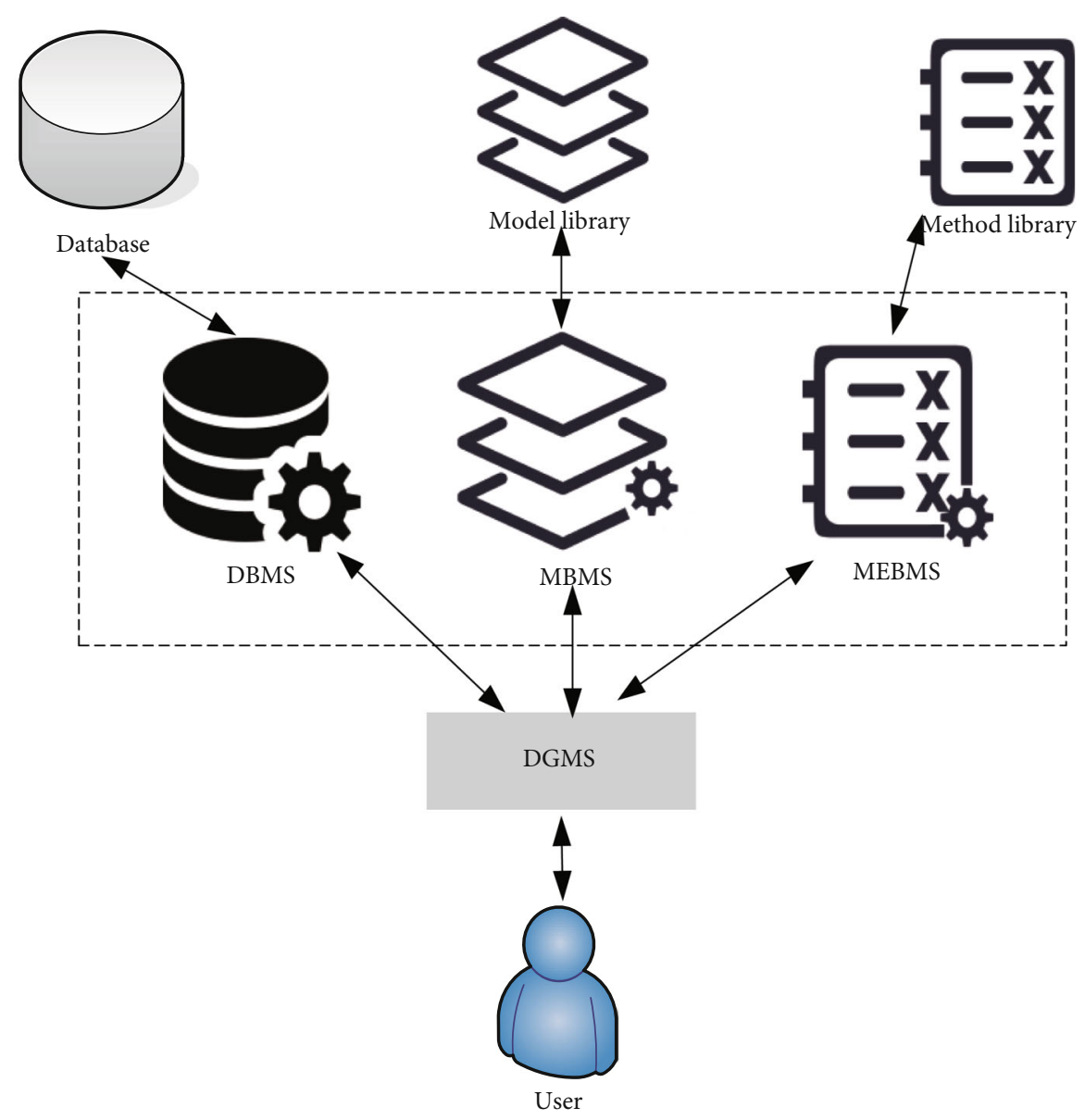

FIgURE 3: Three-database structure of DSS.

In the formula, $\beta_{k}$ is the weight coefficient of each factor and satisfies $\sum_{k=1}^{m} \beta_{k}=1$.

\section{Wireless Communication of Embedded Microprocessor and Computer Vision Referee Decision Support System Test}

3.1. Experimental Environment. The WAMS measurement data were simulated by the transient stability calculation module of the time-domain integration method in the power system analysis integrated program PSASP. The integration step was $0.001 \mathrm{~s}$, the simulation calculation time was $20 \mathrm{~s}$, and the WAMS measurement period was assumed to be $0.01 \mathrm{~s}$. WAMS refers to the use of synchronous phase angle measurement technology to achieve a real-time high rate acquisition of the synchronous phase angle and key grid data by progressively laying out synchronous phase angle measurement units (PMUs) at key measurement points across the network. PSASP is based on the support of a basic grid database, a fixed model library, and a user-defined model library to perform various calculations and analyses of power systems. The support vector machine prediction program was implemented by VC6.0 and MATLAB 6.5 programming, and it was run on a computer with a clock speed of $1.92 \mathrm{GHz}$ and a memory of $256 \mathrm{MB}$ [19]. In addi- tion, the pros and cons of a prediction method were mainly measured from two aspects: prediction accuracy and prediction step size. Therefore, the normalized mean square error, $S_{n r s}$, was defined as the prediction accuracy evaluation index:

$$
S_{n r s}=\frac{1}{S_{o b s}} \sqrt{\frac{1}{M-1} \sum_{k=1}^{M}(x(k)-x \wedge(k))^{2}} .
$$

In the formula, $M$ is the number of predicted points, $S_{o b s}$ is the standard deviation of the actual measurement value, $x(k)$ is the actual measurement value, and $\widehat{x}(k)$ is the predicted value.

3.2. Martial Arts Action Sample Data Set. In the experiment, the mean and variance of the input variables were changed to generate the target data set, $D_{t}$, and multiple source data sets, $D_{s}^{i}$. By adjusting the size of the mean, the similarity between the target field and the source field could be controlled, where each feature variable of $D_{t}$ obeyed the $[0,3]$ Gaussian distribution, the number of training samples generated was 200, and the number of test samples was 100 , by setting the mean value of a feature variable $x_{i}$ to between $[20,30]$, and the variance to $[1,3]$. Then, we generate different source domain data sets. In this experiment, two source fields, $x_{1} \sim[30,2.5]$ and $x_{2} \sim[25,2.7]$, were generated, and the 
number of samples was 1000. To compare the transfer effects of different methods, all samples in the source field were used to assist training, and the number of training samples in the target field was gradually increased for multiple trials [20]. In the data collection stage, sensors could be used to sample martial arts movements to obtain upper limb movement information and lower limb movement information; we performed data preprocessing on the upper limb movement information and lower limb movement information to obtain the preprocessed data information. The upper limb movement information and the lower limb movement information were input into the computer, and the result could be obtained.

3.3. System Function Design. The system is divided into the following parts according to functions, and the functional modules are shown in Figure 4.

(1) Data Preparation Module. Data preparation is divided into three submodules: data cleaning, data conversion, and data extraction. Data cleaning finds errors in data; data conversion converts data from various data sources to the SQL Server 2000 database; data extraction finds the data sets needed for decision support from SQL Server 2000 database

(2) Decision Support Module. All kinds of neural network decision support models and methods were designed to support a reasoning-aided decision

(3) Result Evaluation and Presentation Module. After evaluation, the redundant and irrelevant patterns are eliminated. If the pattern cannot meet the user's requirements, the whole discovery process needs to return to the discovery stage, reselect the data, adopt a new data transformation method, set new data mining parameter values, or change a mining algorithm to recalculate and extract rules. For those valuable and correct results, the information is presented to the user graphically

3.4. Martial Arts Segmentation Modeling. Time segmentation uses the time domain, that is, the information of the preceding and following frames, for segmentation. Because the foreground object usually has a different motion from the background, the approximate area of the moving object can be obtained through change detection. To better extract the target, the time domain changes of the three adjacent frames before, middle, and back are used to detect the moving target to extract the change detection template.

(1) Edge Information Detection. To prevent the influence of local light and dark changes in the image on the target, the Canny edge detection method is used in the time domain change detection method to extract the edge information of the image and eliminate the nonmotion area. The essence of edge detection is to use some algorithm to extract the junction line between the object and the background in an image. We define an edge as the boundary of a region in an image where there is a sharp change in grey level; Canny operator edge detection is a first-order differentiation of a Gaussian function that can strike a good balance between noise suppression and edge detection. The LOG edge detection algorithm can also be used, which first uses bilateral filtering instead of Gaussian filtering, then calculates the Laplacian of the smoothed image, and finally extracts the zero-crossing point after the operation as the edge of the image. This method can better protect the edges, improve detection accuracy, and reduce the number of pseudo-edges

(2) Extract the Initial Segmented Mask Image. To eliminate the influence of background on target extraction, the results of time-domain detection and Canny edge detection are summed to effectively eliminate noise and obtain the initial segmentation mask image. Moreover, morphology is used to correct the image, complete the connection of the fracture edge, and get the final initial segmentation mask image

(3) Target Segmentation. First, the image is reconstructed twice to reduce the noise points in the image as much as possible; second, the image is marked with internal and external markers to mark the foreground target and background; finally, the gradient of the image is calculated, and the gradient image is modified according to the results of the internal and external marking to eliminate the oversegmentation problem caused by the small change of gray level, and the modified gradient image is segmented by watershed. In the process of calculation, there are some noises and small fragments. Morphological and background difference operations are used to optimize the segmentation image. Digital images, in reality, are often affected by noise interference from imaging equipment and the external environment during digitization and transmission and are called noisy images or noise images

\subsection{Model Estimation}

(1) Import Sample Data and Prepare Sample Data. The function prepareDataTrain extracts data from filenamePredictors and returns the unit arrays XTrain and YTrain; it then deletes elements with constant values, normalizes training estimation factors, and normalizes training estimation variables to a zero mean

(2) Define the Network Architecture. Create an LSTM network, which consists of an LSTM layer with 50 hidden units, followed by a fully connected layer with a size of 1 (the number of output variables) and a dropout layer (dropoutlayer) with a loss probability of 0.5 . Set the learning step size (learning rate) to 0.01 , the input node of the input layer to 7 , the hidden layer dimension to 50 , and the output layer to 1 output node. Set the activation function of each 


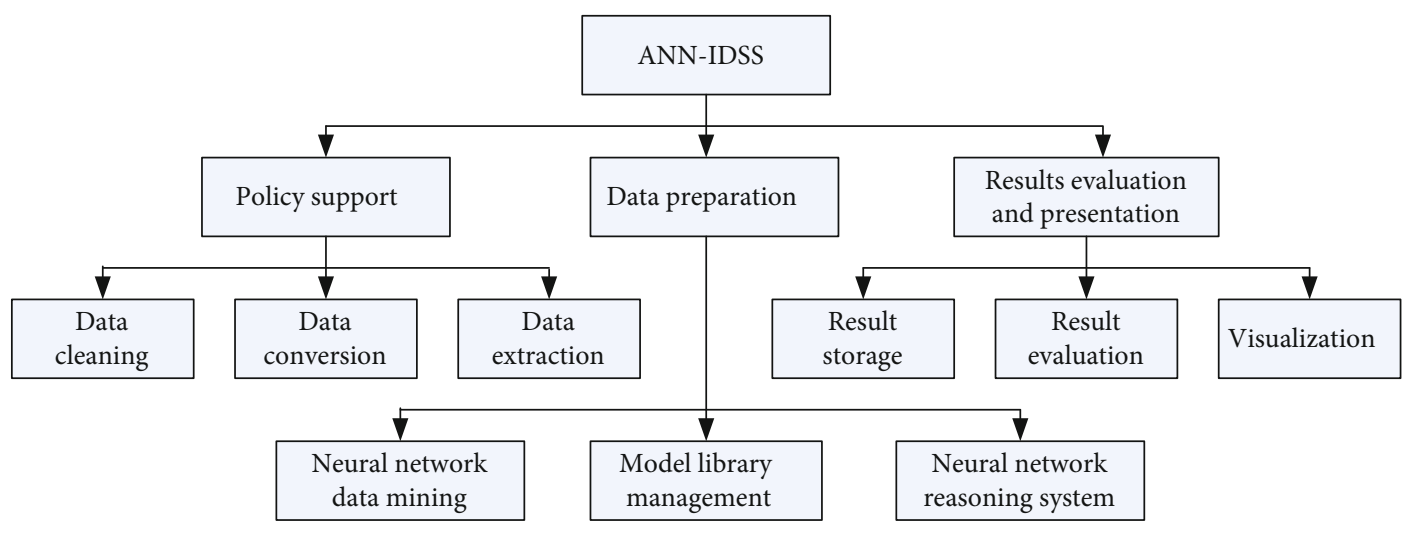

FIGURE 4: Functional modules.

layer to Relu. Set the network optimization algorithm to Adam

(3) Train the Network. Use the function trainNetwork to train the network

(4) Test the Network. The function prepareDataTest extracts data from filenamePredictors and filenameResponses and returns $\mathrm{X}$ Test and Y Test; use predict to estimate the test samples

\section{System Test Results}

4.1. Computer Vision in the System. The reliability of the matching algorithm based on region similarity was affected by the size of the selected support window. The larger the support window, the richer the matching information, and the better the matching effect in the low texture area or repeated area, but the higher the false matching rate in the complex details, disparity discontinuity, or occlusion area. Moreover, the smaller the support window, the less mismatching in the disparity discontinuity area and occlusion area, but the less sufficient the information collection and the higher the false matching rate in the low-texture area. To collect more reference information, the support window had to be enlarged. However, with the increase of the support window, the information of the blocked area in the support window would also increase. The information of the blocked area was invalid or even interference information. The reduction of reference information reliability would directly affect the matching accuracy. The prediction error curve under the condition of gradually increasing sample size in the target domain is shown in Figure 5. It can be seen from the figure that the difference between the predicted value and the real value of mvmo-lssvm is within a reasonable range, while the difference between the predicted value and the real value of molssvm is larger, which indicates that the mvmo-lssvm algorithm is more effective. The molssvm algorithm has a plane selection in its application, and the size of the solution is influenced by the number of samples, resulting in a large solution size. The mvmo-lssvm algorithm starts with a machine learning loss function and uses a twoparameter number in the objective of the optimization problem, enabling the solution of a set of linear equations.
The dots in the graph represent the prediction error curve for a progressively larger sample size in the target domain.

4.2. Decision Support System in Martial Arts Competition. Owing to the times, modern martial arts have been influenced by western culture and developed into martial arts with characteristics of the times, with sports-oriented competitive martial arts being the main content. The marketization of martial arts routines is a product of the market economy system. The establishment of an efficient, fast, and convenient operating, and management mechanisms will play an important and positive role in the spread and development of martial arts. The question of how to establish a brand and win market competitiveness is an important link in the market development of martial arts routines. The length of the time window, the prediction step length, and the prediction accuracy are closely related. The time window means that when the time cycle reaches a certain stage, a turning point will occur. During a certain critical period in the time cycle, if an influence is exerted on a specific thing, or a certain action is taken, the probability of success will increase. Generally speaking, when the time window length is fixed, as the prediction step length increases, the accuracy will decrease. Under the premise of ensuring the prediction accuracy, increasing the large time window length helps to extend the prediction step size. However, if the time window is too long, it will lose the meaning of prediction. Therefore, it is necessary to determine a reasonable time window length and effective prediction step length. For this reason, time windows of different lengths were used in the simulation process to detect the generalization ability of the prediction method. Under different time window lengths, the effective prediction step lengths of a stable trajectory and an unstable trajectory are shown in Table 1 . Under the condition of prediction accuracy $S_{n r s} \leq 0.05$, for the stable trajectory, the ratio of time window length to effective prediction step is close to $2: 1$; for the unstable trajectory, the ratio of time window length to effective prediction step is close to $3: 1$. Considering the requirements of stable trajectory and unstable trajectory prediction, the prediction step length can reach $80-120 \mathrm{~ms}$. As the best method of enterprise data processing in the current situation, the data warehouse is the 


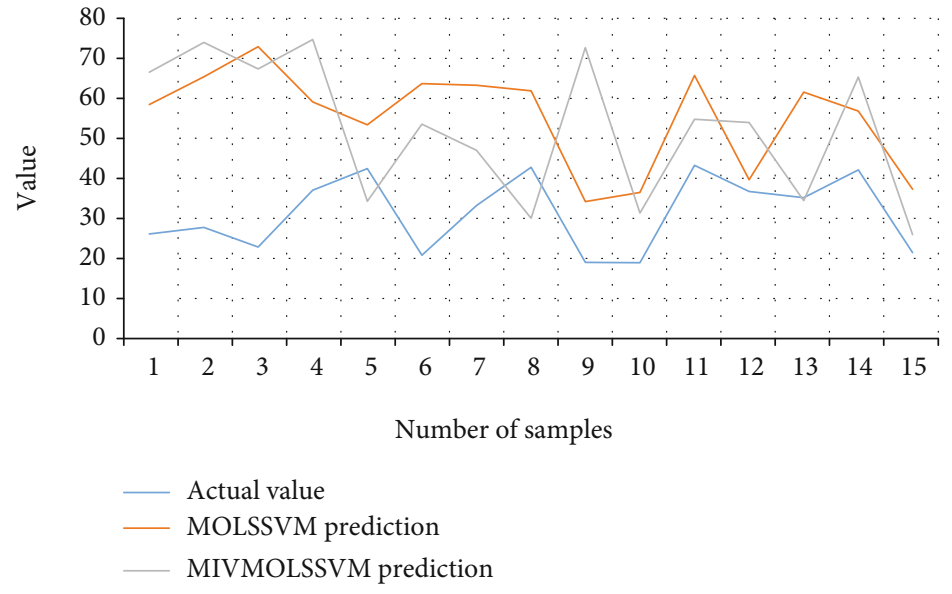

FIGURE 5: Forecast error curve.

TABLE 1: Effective prediction step length for stable trajectory and unstable trajectory.

\begin{tabular}{lcc}
\hline $\begin{array}{l}\text { Track } \\
\text { attributes }\end{array}$ & $\begin{array}{c}\text { Time window } \\
\text { length/ms }\end{array}$ & $\begin{array}{c}\text { Effective prediction step } \\
\text { size/ms }\end{array}$ \\
\hline Stable & 100 & 50 \\
Stable & 200 & 90 \\
Stable & 300 & 140 \\
Instability & 100 & 40 \\
Instability & 200 & 80 \\
Instability & 300 & 120 \\
\hline
\end{tabular}

TABLE 2: Test results.

\begin{tabular}{lccc}
\hline $\begin{array}{l}\text { Serial } \\
\text { number }\end{array}$ & $\begin{array}{c}\text { Detect the number of } \\
\text { pedestrians }\end{array}$ & $\begin{array}{c}\text { The actual number } \\
\text { of pedestrians }\end{array}$ & Accuracy \\
\hline 1 & 16 & 14 & $87.50 \%$ \\
2 & 15 & 16 & $93.70 \%$ \\
3 & 17 & 16 & $94.10 \%$ \\
4 & 18 & 15 & $83.30 \%$ \\
5 & 18 & 16 & $88.80 \%$ \\
6 & 17 & 17 & $100.00 \%$ \\
7 & 16 & 20 & $80.00 \%$ \\
8 & 17 & 21 & $80.90 \%$ \\
9 & 19 & 20 & $95.00 \%$ \\
10 & 16 & 17 & $94.10 \%$ \\
11 & 17 & 19 & $89.40 \%$ \\
12 & 17 & 16 & $94.10 \%$ \\
13 & 20 & 17 & $85.00 \%$ \\
\hline
\end{tabular}

product of the combination of multiple disciplines. Resource management and data storage were realized through traditional database technology, which is also the basic method of data warehouse technology. Information extraction and data analysis were realized by using statistical analysis technology. Of course, this is also the most effective method. The mining of knowledge and the exploration of laws were achieved by using artificial intelligence technology, which is also the most scientific way at present.

Through video detection, the connected domain analysis was carried out, the number of targets was counted, the number of people in the current video was obtained, and the statistics were written into the database in real-time. The test results are shown in Table 2 and Figure 6. It can be seen that except for the low-recognition accuracy rate in individual cases, the detection accuracy rate of most pedestrian numbers is relatively high, the accuracy rate is between $70 \%$ and $100 \%$, and the effect is relatively ideal. At the same time, through statistics, the algorithm takes an average of $0.2 \mathrm{~s}$ to process a frame of an image. Through sampling at reasonable intervals, it can meet the real-time requirements. When the error distance between the center point of the predicted target tracking frame and the center point of the true value is less than 12 pixels, the SCM algorithm and the ASLA algorithm are slightly better than our DPL algorithm, but when the distance error gradually increases. The target tracking algorithm shows a more stable performance, surpassing all target tracking algorithms. This also shows from the side that the target tracking algorithm that integrates deep learning and preference learning rarely loses the target. In other words, the algorithm can handle various interference factors from the target tracking process. The SCM algorithm refers to a deformable curve defined in the image domain, and by minimizing its energy function, the dynamic profile gradually adjusts its shape to match the target profile. The ASLA algorithm refers to a tracking method based on feature matching that does not consider the overall characteristics of the moving target but only tracks through some salient features of the target image.

The percentages in Figure 6 represent the accuracy, and the data mainly express that the detection accuracy is high in most cases, except for a few cases where the recognition accuracy is low, and the accuracy is between $70 \%$ and $100 \%$, which is more satisfactory.

4.3. Target Detection Algorithm. The comparison of algorithm extraction results is shown in Figure 7. It can be seen from the figure that the method in this paper can achieve the 


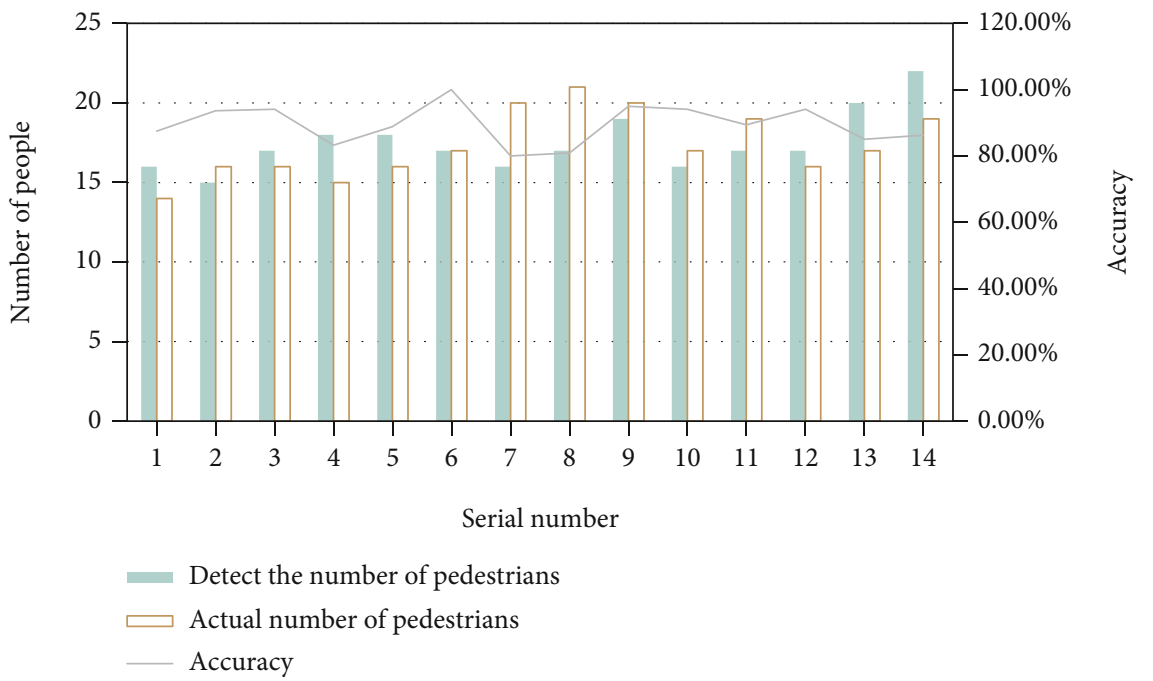

Figure 6: Test results.

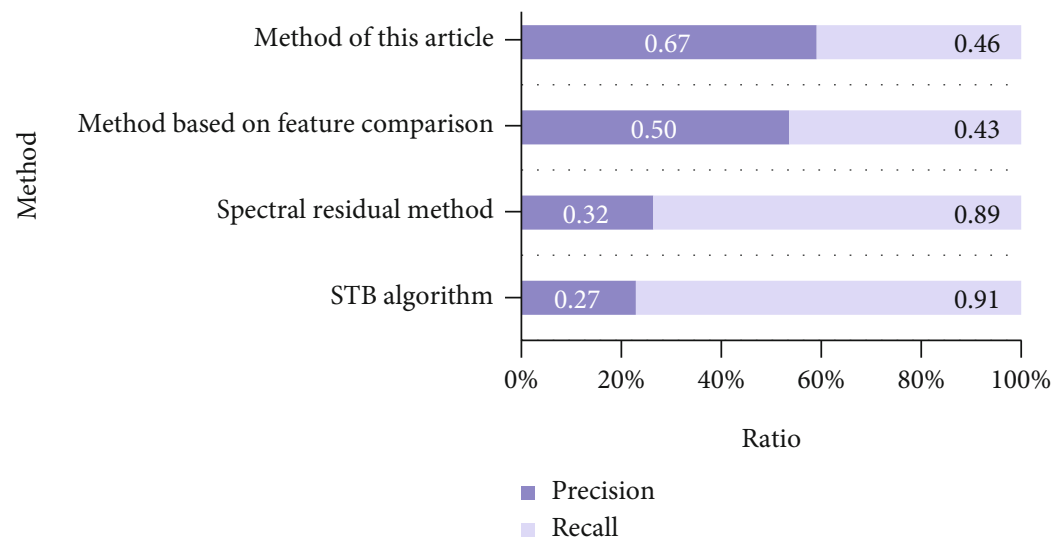

FIgURE 7: Algorithm extraction results.

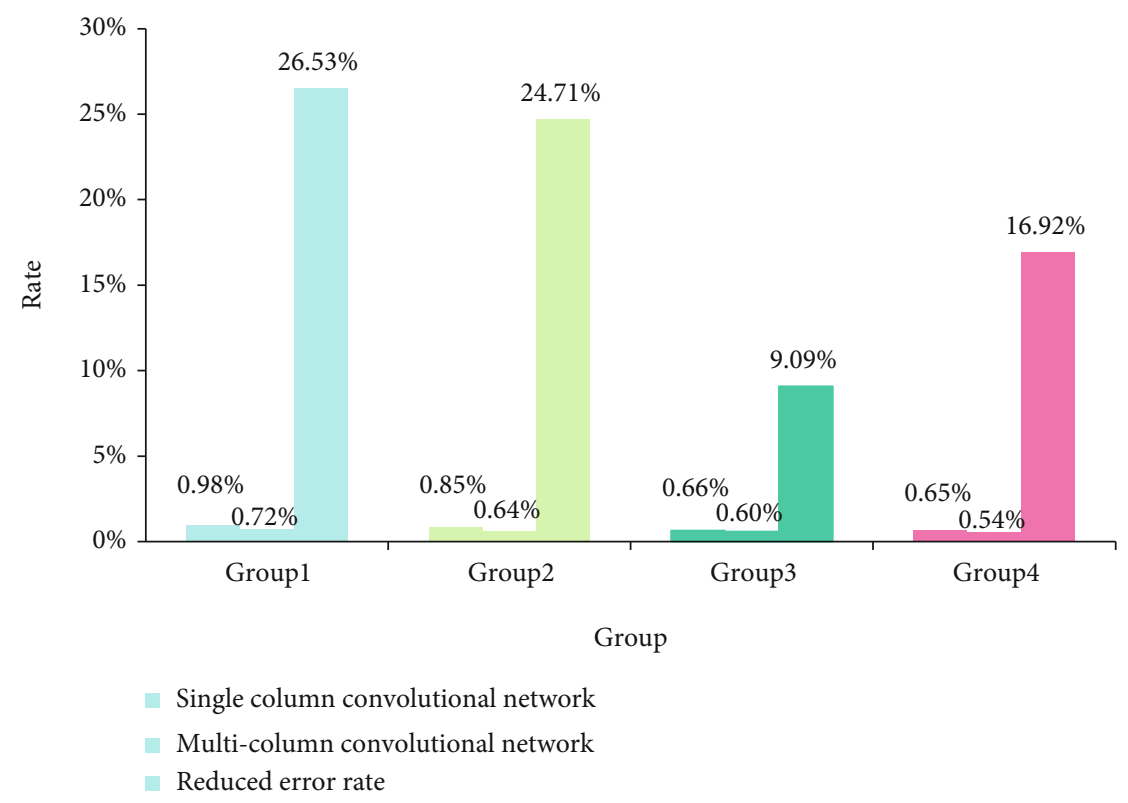

FIGURE 8: Error rate test results. 


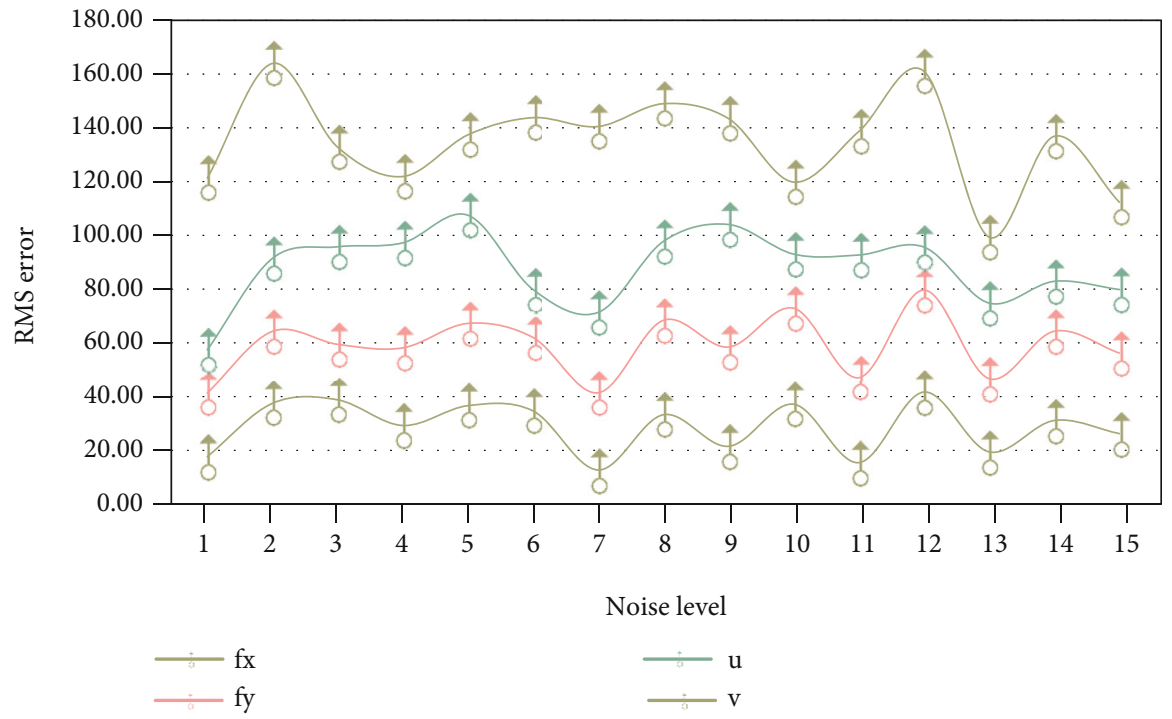

Figure 9: Errors of calibration results under different noise levels.

best results on four different data sets, and the salient areas obtained can correctly point to the foreground target. The STB method has a higher reproducibility rate than the accuracy rate. Because the size and range of the salient target cannot be determined, although the saliency map generated by the STB method can get the position of the salient target, it often contains a large number of background areas. The method based on feature comparison also has limitations. When the feature of the current scene target is close to the average feature of the image, the method based on feature comparison cannot identify the foreground target and the background area, and the saliency map obtained cannot point to the foreground target correctly. The spectral residual method mainly focuses on the parts where the image features change drastically. Therefore, the spectral residual method has a richer texture and a better extraction effect for more obvious images and extracts a saliency map for images that are not rich in texture and contrast. The effect is poor, so the result of the spectral residual method is the worst among the four methods.

As the depth of the neural network deepens, its model capacity will gradually increase. However, when the neural network develops to a certain depth, the performance will decrease instead. This is because after the depth continues to increase, the gradient in the back propagation process will also increase or decrease drastically, which will cause the gradient to disappear or explode, which is not conducive to the optimization of the neural network. The same problem exists in cyclic neural networks. In backpropagation, for the earlier propagation process, the gradient of its weight is prone to disappear or explode. The error rate test results of multicolumn convolutional neural networks based on different convolution kernel sizes on the MNIST data set are shown in Figure 8. In Group 1 and Group 2, the error rate of the multicolumn convolutional neural network fused by sliding window is about $25 \%$ lower than that of the singlecolumn convolutional neural network. Group 3, as the group with the smallest drop in the error rate, also achieves a $9 \%$ drop in the error rate. This result shows that by constructing multicolumn convolutional neural networks with different convolution kernel sizes, combined with the sliding window fusion algorithm, it is possible to achieve better object recognition accuracy than single-column convolutional neural networks.

4.4. Algorithm Error Analysis. The error of the calibration results under different noise levels is shown in Figure 9. When the noise level is small, the error of the calibration result of the algorithm in this paper is usually small, and this error increases with the increase of noise. Although the result of the skew factor is not shown in the figure, it has the same properties. When the number of line segments used in the algorithm increases, the robustness of the algorithm improves. The calibration results obtained using 50 line segments are significantly better than the calibration results obtained using 25 line segments.

\section{Conclusions}

This paper mainly studied the application of wireless communication with an embedded microprocessor and computer vision in the decision support system for referees in martial arts competitions. Computer vision technology has penetrated and plays an important role in many aspects of scientific research and life. When the target enters the scene, the moving target detection method is used to calibrate the tracking target, and the template is initialized to realize automatic selection of the tracking target template. By combining value segmentation technology with the phase consistency detection algorithm and through the image preprocessing method of the embedded microprocessor, the detection area was reduced. Moreover, not only has the advantage of high calculation efficiency of the threshold segmentation algorithm but also the ability to process the signal, voice, and image, the transmission speed was fast, the transmission was stable, and it had good robustness. 
Combining the basic business knowledge of martial arts competitions, the intelligent decision support system constructed in this paper is required to analyze the needs. The content mainly includes system business demand analysis, functional demand analysis, and data demand analysis. In the inherent decision support system, the general model is as follows: first, a decision model is established, and then, the model predicts future affairs through an in-depth analysis and calculation of the data in the existing database, and finally, it gives effective information to the decision-maker. We establish DW and use OLAP and DM tools to display and analyze data and provide support for decision-making. This is a decision support system in a sense.

The standardization of martial arts equipment can enable manufacturers of martial arts equipment to realize the pressure of competition and actively invest $\mathrm{R} \& \mathrm{D}$ funds for technological innovation and production technology innovation, to eliminate outdated production capacity and low-qualified manufacturers that do not meet the standards, and to change the original production model and marketing concept. It can also help those who actively seek high-tech materials to upgrade martial arts equipment, reduce damage to the environment, and promote the healthy and sustainable development of the entire sports industry. Using this evaluation method, the images of the salient areas of the scene extracted from the multiscale are subjected to the feature extraction of the convolutional neural network to obtain their feature expression. Finally, the features of various scales are fused to characterize the features of the entire scene and complete the scene recognition.

\section{Data Availability}

The data that support the findings of this study are available from the corresponding author upon reasonable request.

\section{Conflicts of Interest}

The authors declare that they have no conflicts of interest.

\section{Authors' Contributions}

Jingjing Ji and Feng Liang contributed equally to this work as co-first author.

\section{Acknowledgments}

We thank LetPub (http://www.letpub.com/) for its linguistic assistance during the preparation of this manuscript. This work is supported by the Thousands of young and middle-aged backbone teachers in Guangxi colleges and universities training plan, humanities and social sciences project "Guangxi Community Sports Public Facilities Management Service Outsourcing Theory and Empirical Research' (No. 2021QGRW065).

\section{References}

[1] F. Tarlak, M. Ozdemir, and M. Melikoglu, "Computer vision system approach in colour measurements of foods: part I.
Development of methodology," Science and Technology, vol. 36, no. 2, pp. 382-388, 2016.

[2] L. Fusco, R. Lefort, K. Smith et al., "Computer vision profiling of neurite outgrowth dynamics reveals spatiotemporal modularity of Rho GTPase signaling," The Journal of Cell Biology, vol. 212, no. 1, pp. 91-111, 2016.

[3] N. Naik, R. Raskar, and C. A. Hidalgo, "Cities are physical too: using computer vision to measure the quality and impact of urban appearance," American Economic Review, vol. 106, no. 5, pp. 128-132, 2016.

[4] J. Wäldchen and P. Mäder, "Plant species identification using computer vision techniques: a systematic literature review," Archives of Computational Methods in Engineering, vol. 25, no. 2, pp. 507-543, 2018.

[5] S. Zhou, M. Ke, and P. Luo, "Multi-camera transfer GAN for person re-identification," Journal of Visual Communication and Image Representation, vol. 59, pp. 393-400, 2019.

[6] K. Gopalakrishnan, S. K. Khaitan, A. Choudhary, and A. Agrawal, "Deep Convolutional Neural Networks with transfer learning for computer vision- based data-driven pavement distress detection," Construction and Building Materials, vol. 157, no. 12, pp. 322-330, 2017.

[7] H. Hamledari, B. Mccabe, and S. Davari, "Automated computer vision-based detection of components of underconstruction indoor partitions," Automation in Construction, vol. 74, no. 2, pp. 78-94, 2017.

[8] T. B. Moeslund, G. Thomas, A. Hilton, P. Carr, and I. Essa, "Computer vision in sports," Computer Vision and Image Understanding, vol. 159, no. 6, pp. 1-2, 2017.

[9] A. R. di Rosa, F. Leone, F. Cheli, and V. Chiofalo, "Fusion of electronic nose, electronic tongue and computer vision for animal source food authentication and quality assessment - a review," Journal of Food Engineering, vol. 210, no. 10, pp. 62-75, 2017.

[10] D. Feng and M. Q. Feng, "Computer vision for SHM of civil infrastructure: from dynamic response measurement to damage detection - a review," Engineering Structures, vol. 156, no. 2, pp. 105-117, 2018.

[11] S. Yeung, N. L. Downing, L. Fei-Fei, and A. Milstein, "Bedside computer vision-moving artificial intelligence from driver assistance to patient safety," New England Journal of Medicine, vol. 378, no. 14, pp. 1271-1273, 2018.

[12] H. Scharr, H. Dee, A. P. French, and S. A. Tsaftaris, "Special issue on computer vision and image analysis in plant phenotyping," Machine Vision and Applications, vol. 27, no. 5, pp. 607-609, 2016.

[13] T. Hoyoux, A. J. Rodriguez-Sanchez, and J. H. Piater, "Can computer vision problems benefit from structured hierarchical classification?," Machine Vision and Applications, vol. 27, no. 8, pp. 1299-1312, 2016.

[14] C.-w. Dong, H.-k. Zhu, J.-w. Zhao, Y.-w. Jiang, H.-b. Yuan, and Q.-s. Chen, "Sensory quality evaluation for appearance of needle-shaped green tea based on computer vision and nonlinear tools," Journal of Zhejiang University-SCIENCE B, vol. 18 , no. 6 , pp. 544-548, 2017.

[15] Z. Lv, A. Halawani, S. Feng, S. ur Réhman, and H. Li, “Touchless interactive augmented reality game on vision-based wearable device," Personal and Ubiquitous Computing, vol. 19, no. 3-4, pp. 551-567, 2015.

[16] S. Y. Du and Z. G. Liu, "A comparative study of different color spaces in computer-vision-based flame detection," 
Multimedia Tools and Applications, vol. 75, no. 17, pp. 10291-10310, 2016.

[17] N. A. Khan, H. Pervaz, A. Latif, and A. Musharaff, "Report: unsupervised identification of malaria parasites using computer vision," Pakistan Journal of Pharmaceutical Sciences, vol. 30, no. 1, pp. 223-227, 2017.

[18] M. Tretola, A. R. di Rosa, E. Tirloni et al., "Former food products safety: microbiological quality and computer vision evaluation of packaging remnants contamination," Food Additives \& Contaminants, vol. 34, no. 8, pp. 1427-1435, 2017.

[19] S. Ding, S. Qu, Y. Xi, and S. Wan, "Stimulus-driven and concept-driven analysis for image caption generation," Neurocomputing, vol. 398, pp. 520-530, 2020.

[20] D. M. Alemayehu, A. D. Mengistu, and S. G. Mengistu, "Computer vision for Ethiopian agricultural crop pest identification," Indonesian Journal of Electrical Engineering and Computer Science, vol. 3, no. 1, pp. 209-214, 2016. 